\title{
Raman Spectra of Passive Films of Iron in Neutral Borate Solution
}

\author{
Toshiaki Ohtsuka \\ Department of Applied Chemistry, Nagoya Institute of Technology, Gokiso-cho, Showa-ku, \\ Nagoya 466, Japan
}

\begin{abstract}
The Raman spectra of the passive films formed on iron in a neutral borate solution at various potentials were measured for evaluating its composition without any enhancement technique. The ex-situ spectrum measured in air after removal from the aqueous solution corresponds to the composition of disordered $\mathrm{Fe}_{3} \mathrm{O}_{4}$, i.e., $\mathrm{Fe}_{3-\delta} \mathrm{O}_{4}$. The passive film in the aqueous solution, which could not be measured in this paper due to a large background scattering light intensity from surrounding water around the iron electrode, may be a compositional framework same as the ex-situ passive film, although it is expected to be hydrated under the in-situ condition. For the in-situ measurement of the passive film, it is speculated that much accumulation will be required to decrease the noise level of the background light scattering relative to the signal from the thin film.
\end{abstract}

(Received September 12, 1995)

Keywords: iron, passive film, oxide, Raman spectroscopy, borate solution

\section{Introduction}

The composition of iron passive film has been discussed by various authors. From the results obtained the film has been suggested to consist of a $\mathrm{Fe}(\mathrm{III})$ oxyhydroxide containing some amount of water. O'Grady proposed from the in-situ Mössbauer spectra of the film a polymeric structure of a hydrated $\mathrm{Fe}(\mathrm{III})$ oxide that contains $\mathrm{OH}$ bridge $^{(1)}$. Brett et al. evaluated from the Mössbauer spectra the amorphous Fe(III) oxy-hydroxide $^{(2)}$. From the X-ray absorption experiments that were conducted by Kruger et al., the amorphous and hydrated $\mathrm{Fe}(\mathrm{III})$ oxy-hydroxide was also considered ${ }^{(3)-(5)}$.

Rubim et al. ${ }^{(6)}$, Guy et al. ${ }^{(7)-(9)}$, and Melendres et al. ${ }^{(10)}$ applied the surface enhanced Raman scattering (SERS) spectroscopy to the passive film on iron. They measured the Raman spectra of the passive film formed on iron on which Ag particles were deposited for enhancing the SERS sensitivity. They claimed that the Ag particles were not affected on the behavior of the passive iron; however, the current-potential curve reported for the iron electrode is not conceivable to correspond to that of pure iron. They found the presence of $\mathrm{Fe}(\mathrm{OH})_{2}$ in the film formed in the potential region less noble than the Flade potential. They further estimated that the film in the passive potential region may consist of $\mathrm{Fe}_{3} \mathrm{O}_{4}$ and $\mathrm{FeOOH}$, but the SERS spectra from the film were not enough correspondent to those of the crystalline compounds. In the SERS measurement of the passive film, the problems should be discussed whether the Ag particle overlayer deposited on the iron influences the properties of the passive films and whether the Raman spectra are changed by the Ag particles. A comparison of the SERS spectra with the normal Raman spectra will be necessary for the further discussion.
With the normal Raman spectroscopy (NRS), since the ordinary passive film is too thin to be measured under the in-situ condition, the potential cycles were attempted between hydrogen evolution potential region and passive potential region in order to obtain a thick film enough to be detected by $\operatorname{NRS}^{(11)(12)}$. The potential cycles induce a redox reaction between $\mathrm{Fe}$ (III) and $\mathrm{Fe}$ (II) in the film in addition to the film formation, and the thick film obtained after the potential cycles may thus be different in composition from the ordinary film.

In this paper we at first tried the measurement of the ordinary passive film by NRS. However, we failed in obtaining the Raman spectrum of the passive film under the in-situ condition and then measured it under the ex-situ condition in air. We, therefore, reported the normal Raman spectra in air after the polarization in the aqueous solution and discuss the possibility of obtaining the normal Raman spectra of the passive film under the in-situ condition.

\section{Experimental}

The iron electrode used was prepared from a sheet 1 mm thick of $99.99 \%$ pure Ferrvac-E iron. The electrode surface was mechanically polished by $0.05 \mu \mathrm{m}$ alumina abrasive and ultrasonically washed in acetone. The solution was a 1:1 mixture of $0.075 \mathrm{M}\left(\mathrm{mol} \mathrm{dm}^{-3}\right) \mathrm{Na}_{2} \mathrm{~B}_{4} \mathrm{O}_{7}$ and $0.3 \mathrm{M} \mathrm{H}_{3} \mathrm{BO}_{3}$ at $\mathrm{pH} 8.4$, being prepared from analytically grade reagents and pure water obtained by de-ionization and subsequent distillation in a quartz vessel.

The Raman spectrometer used was a triple type of spectrometer (JASCO R-800T) consisting of a double monochromator (80-cm light-path length) and a third monochromator (40-cm light-path length). The band path width of the monochromator in the present measurement was about $8 \mathrm{~cm}^{-1}$. A single line of argon ion laser at 
the wavelength of $514.5 \mathrm{~nm}$ was used for excitation of the Raman scattering. The laser light for the excitation was polarized in the parallel direction to the incidence plane and its incidence angle was about $50^{\circ}$. The Raman scattering light was collected in the incidence plane in the direction normal to the incident light.

\section{Results and Discussion}

In this study we at first measured Raman spectra of the passive films in the neutral borate solution under the insitu condition by NRS with an optical cell described in the earlier paper ${ }^{(13)}$. In the experiment, the potential was held at constant in the solution for $1 \mathrm{~h}$ before the NRS measurement. We obtained no Raman spectra from the film, but only the spectra from water and borate anions. The Raman scattering efficiency from the surface thin film may be too small, if being compared with that from the aqueous solution surrounding the electrode surface at the focussed area of the laser light. In this paper we thereafter report the ex-situ Raman spectra of the passive film in air after removal from the aqueous solution and we will discuss a possibility of obtaining the Raman spectra from the film under the in-situ condition in aqueous solution.

Figure 1 shows the ex-situ Raman spectra of the passive film which was formed by constant potentials for $1 \mathrm{~h}$ in the borate solution. The Raman bands are not noticeable: however, a main band around $670 \mathrm{~cm}^{-1}$ can be seen which tends to increase in intensity with potential increase. This band becomes broad in the oxygen evolution potential region higher than $1 \mathrm{~V}$. The small faint bands can be seen around $300 \mathrm{~cm}^{-1}$ and $550 \mathrm{~cm}^{-1}$, which become noticeable at the high potentials in the oxygen evolution region. We measured Raman spectra of various $\mathrm{Fe}$ oxides and hydroxides $\left(\alpha-\mathrm{Fe}_{2} \mathrm{O}_{3}, \alpha-\mathrm{Fe}_{2} \mathrm{O}_{3}, \mathrm{Fe}_{3} \mathrm{O}_{4}\right.$, $\alpha-\mathrm{FeOOH}, \beta-\mathrm{FeOOH}$, and $\gamma-\mathrm{FeOOH})$ for references. The spectra are shown in Figs. 2 and 3. For the measurement of Raman spectra of the standard oxide and hydroxide samples the thin layer of each Fe oxide and hydroxide dispersed in $\mathrm{KBr}$ was formed on a stainless steel sheet ${ }^{(14)}$. The samples of the iron oxides $\left(\alpha-\mathrm{Fe}_{2} \mathrm{O}_{3}, \gamma-\mathrm{Fe}_{2} \mathrm{O}_{3}\right.$, and $\left.\mathrm{Fe}_{3} \mathrm{O}_{4}\right)$ and the hydroxide $(\alpha-\mathrm{FeOOH})$ were purchased by Rare Metallic Co. LTD, Tokyo. The other hydroxide samples were prepared in the similar way as described by Schwertmann and Cornell ${ }^{(15)}$. The Raman spectra of the Fe oxide and hydroxide samples were measured under the same geometric condition as that of the passive film. The Raman band positions for the passive film under the exsitu condition disagree with those of any $\mathrm{Fe}$ hydroxide. The main Raman band of the passive film corresponds to a large band of $\mathrm{Fe}_{3} \mathrm{O}_{4}$ at $675 \mathrm{~cm}^{-1}$ and the other band positions are also similar to those of $\mathrm{Fe}_{3} \mathrm{O}_{4}$, although the Raman spectra of the passive film are not clear. The intensity of the sub-bands at 550 and $300 \mathrm{~cm}^{-1}$ of $\mathrm{Fe}_{3} \mathrm{O}_{4}$ appear to depend on samples of $\mathrm{Fe}_{3} \mathrm{O}_{4}$. These sub-bands may be an indicator of disorder of $\mathrm{Fe}_{3} \mathrm{O}_{4}$; the intensity increase may corresponds to a change of nonstoichiometry from $\mathrm{Fe}_{3} \mathrm{O}_{4}$ to $\mathrm{Fe}_{3-\delta} \mathrm{O}_{4}$.
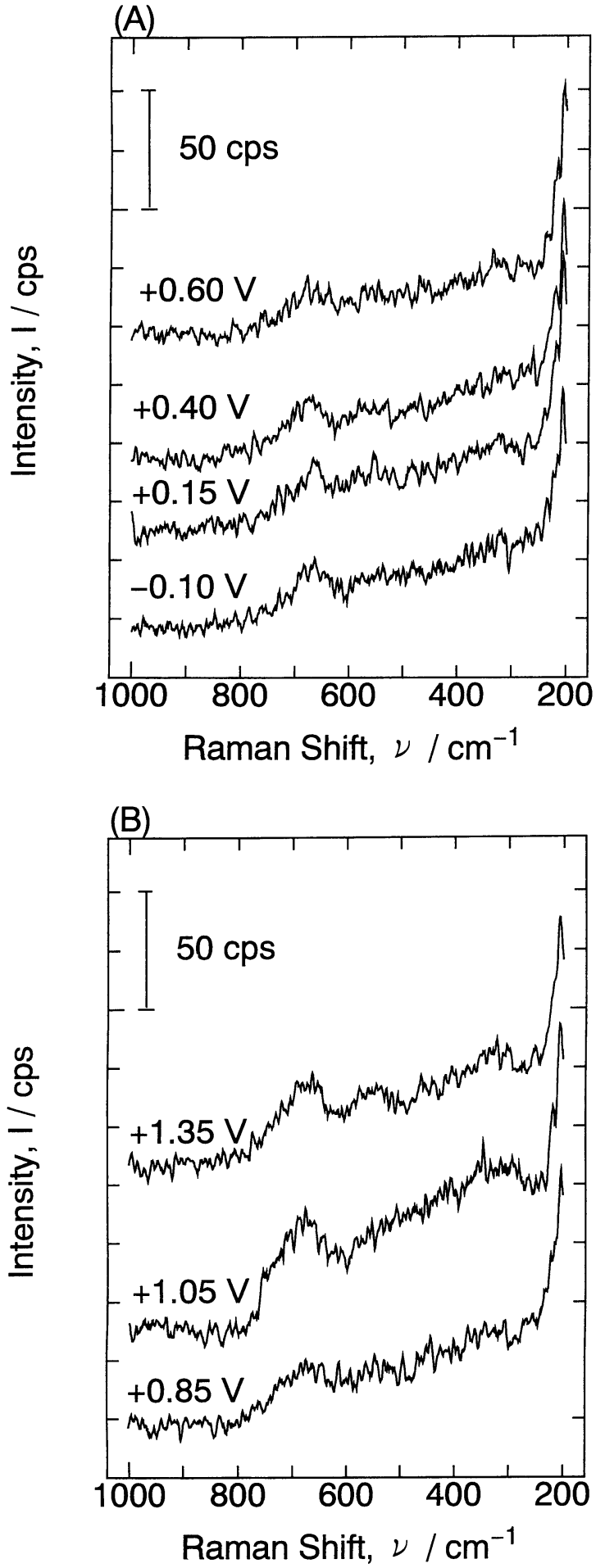

Fig. 1 Raman spectra of passive films on iron under the ex-situ condition. The film was formed by $1 \mathrm{~h}$ oxidation at constant potentials in neutral borate solution at $\mathrm{pH} 8.4$. (A): $-0.10 \mathrm{~V} \sim+0.60 \mathrm{~V}$ (vs. Ag$\mathrm{AgCl})$. (B): $+0.85 \mathrm{~V} \sim+1.35 \mathrm{~V}$.

Since the spectra reported here for the passive film are obtained under the ex-situ condition, a possibility of composition change from the film in aqueous solution can not be excluded. O'Grady pointed out from Mössbauer spectroscopy that the film changes from a hydrated form in the aqueous solution to $\gamma-\mathrm{Fe}_{2} \mathrm{O}_{3}$ in air during drying ${ }^{(1)}$. We, however, believe that the compositional framework 


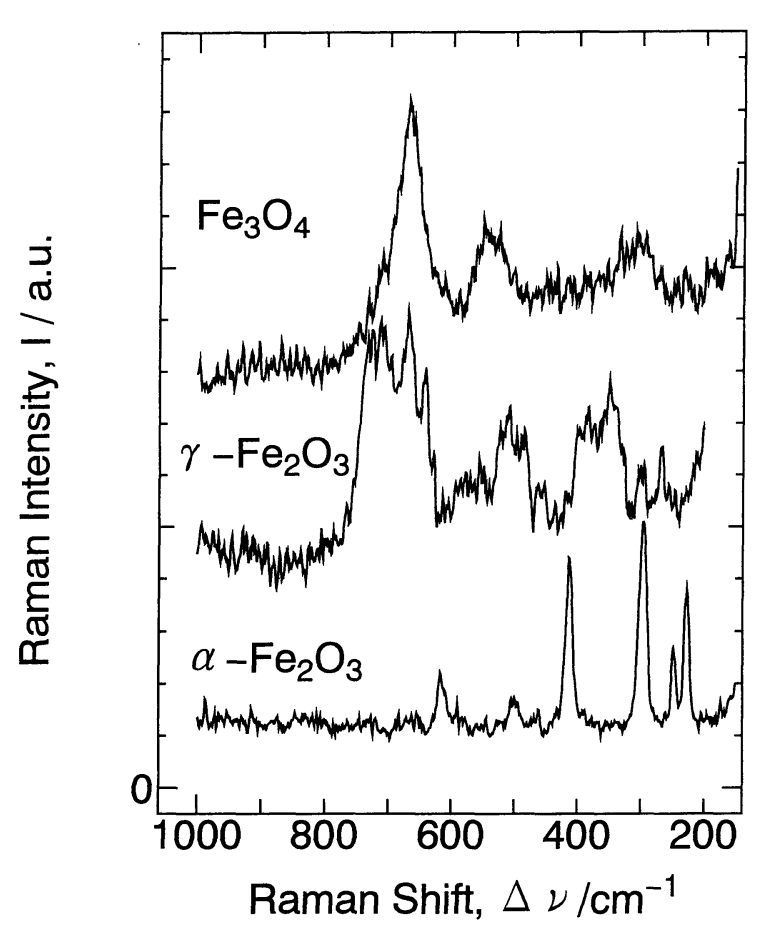

Fig. 2 Raman spectra of Fe oxides. The spectra were measured with thin layer samples dispersed in $\mathrm{KBr}$ on stainless sheet.

may not change dramatically and we suppose that the film consists of a hydrated form of $\mathrm{Fe}_{3} \mathrm{O}_{4}-\gamma-\mathrm{Fe}_{2} \mathrm{O}_{3}$ or $\mathrm{Fe}_{3-\delta} \mathrm{O}_{4}$ in aqueous solution.

In-situ measurement is desirable to characterize the passive films. NRS can not sensitively detect the passive film under the in-situ condition and then an enhanced technique should be introduced. However, we can not believe that the Ag particles deposited on iron for the SERS measurement do not affect the composition of the passive film on iron. It should be preferred that the sensitivity of the Raman spectrum measurement is increased in order to measure the passive film with NRS. With the optical cell used in this study ${ }^{(13)}$, we obtained a scattering light signal about $2000-3000 \mathrm{cps}$ in photon counting rate from the borate solution surrounding the electrode at focussed light area. From the ex-situ measurement of the passive film in air we obtained a peak signal about $20-30 \mathrm{cps}$, as having been described in Fig. 1. The signal from the passive film is 1/100 time as small as the background from the solution. In order to obtain a distinguishable signal from the passive film under the in-situ condition, much accumulation of the signal is required. In this paper we used a single channel detection for the Raman measurement, and, however, the multi-channel detection or the FT-Raman detection will, however, possibly catch the Raman signal of the thin passive film under the in-situ condition.

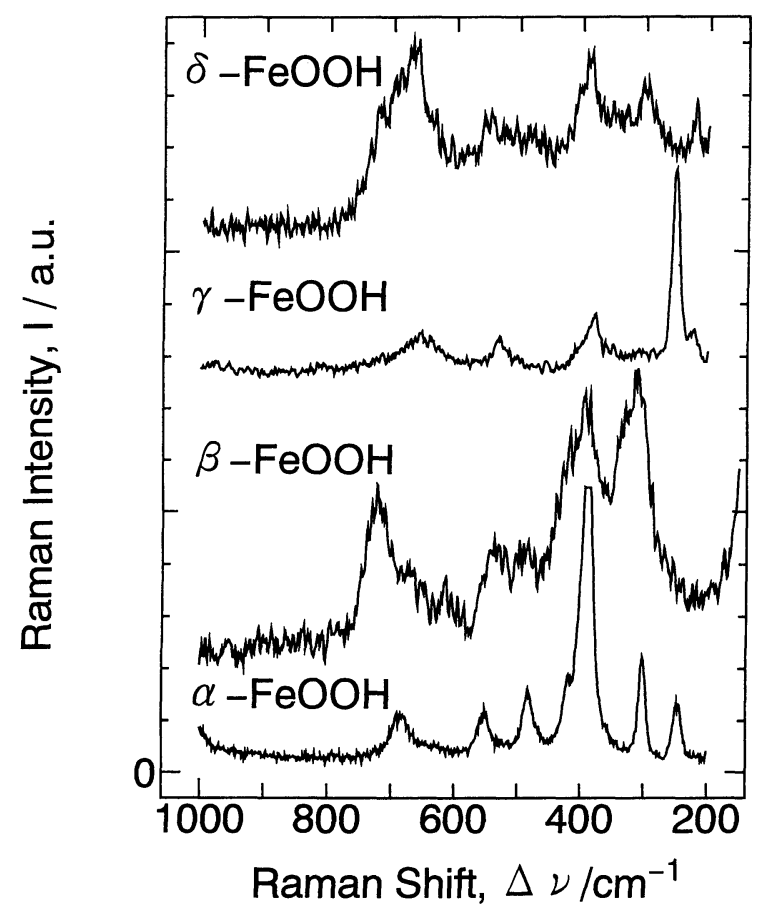

Fig. 3 Raman spectra of Fe hydroxides. The spectra were measured with thin layer samples dispersed in $\mathrm{KBr}$ on stainless sheet.

\section{REFERENCES}

(1) W. E. O'Grady: J. Electrochem. Soc., 155 (1980), 555.

(2) M. E. Brett, K. M. Parkin and M. Graham: J. Electrochem. Soc., 133 (1986), 2031.

(3) J. Kruger: Corros. Sci., 29 (1989), 149.

(4) G. G. Long, J. Kruger, D. R. Black and M. Kuriyama: J. Electrochem. Soc., 130 (1983), 240.

(5) G. G. Long, J. Kruger, M. Kuriyama, D. R. Black, E. N. Fababaugh, D. M. Saunders and A. I. Goldman: Proc. Intern. on Metallic Corrosion, National Council Canada, Ottawa, Vol. 3 (1984), p. 419.

(6) J. C. Rubim and J. Dunwald: J. Electroanal. Chem., 258 (1989), 327.

(7) J. Gui and T. M. Devine: Corros. Sci., 32 (1991), 1105.

(8) J. Gui and T. M. Devine: J. Electrochem. Soc., 138 (1991), 1376.

(9) J. Gui and T. M. Devine: Corros. Sci., 36 (1994), 441.

(10) C. A. Melendres, M. Pankuch, Y. S. Li and R. L. Knight: Electrochim. Acta, 37 (1992), 2747.

(11) J. Dunwald, R. Lossy and A. Otto: Passivity of Metals and Semiconductors, Ed. by M. Froment, Elsevier, Amsterdam, (1983), p. 107.

(12) N. Boucherit, A. Hugot-Le Goff and S. Joiret: Corros. Sci., 32 (1991), 497.

(13) T. Ohtsuka, J. Guo and N. Sato: J. Electrochem. Soc., 133 (1986), 2473.

(14) T. Ohtsuka, K. Kubo and N. Sato: Corrosion, 42 (1986), 476.

(15) U. Schwertmann and R. M. Cornell: Iron Oxides in the Laboratory, VCV, Weinheim, Germany (1991), p. 85, p. 96 and p. 117. 